\title{
Fonctionnement interlingual des mèmes humoristiques : entre création, interprétation et traduction
}

\author{
Interlingual operation of humorous memes: \\ between creation, interpretation and translation
}

\author{
Magdalena Szeflińska-Baran \\ Université de Łódź \\ magdalena.szeflinska@uni.lodz.pl
}

\begin{abstract}
The article focuses on the multisemiotic functioning of Internet memes in communication through the web, focused, among other things, on a humorous effect. The role of the image, first of all, in the creation of the Internet meme and also in its re-application in a multicultural and interlingual environment seems fundamental. This iconic element is part of the large and varied number of relationships with other types of signs (linguistic, cultural, discursive). It seems that the question of the typological diversity of image-text relations (in the very broad sense of it) can be addressed from a variety of perspectives that involve not only a philosophy of translation, but also an approach to humorous communication on the Internet. The article aims to analyse the nature of the relationship that unites an iconic element with a linguistic element that constitutes the essence of the message conveyed by internet memes.
\end{abstract}

Keywords: Internet memes, intersemiotic translation, humorous communication

\section{INTRODUCTION}

L'article se concentre sur le fonctionnement multisémiotique des mèmes Internet dans la communication à travers le web, focalisée, entre autres, sur un effet humoristique. Le rôle de l'image, tout d'abord, dans la création du mème Internet et aussi dans sa réduplication dans un milieu multiculturel et interlingual semble fondamental. Cet élément iconique y entre dans un nombre important et varié de relations avec 
d'autres types de signes : linguistiques, culturels et discursifs. La question de la diversité typologique des relations image-texte (au sens très large de celui-ci) se laisse analyser à partir des optiques variées qui impliquent non seulement une philosophie de la traduction, mais aussi une approche de la communication humoristique sur Internet. Étant donné que cet humour, enfermé entre image et texte, appartient plutôt au domaine de l'implicite discursif susceptible d'influencer le sens du message transmis au sein d'une même langue, il semble justifié d'appliquer à l'analyse traductologique de ce phénomène une approche pragmatique étudiant sa praxis dans le contexte interlingual de la cyberculture. Premièrement, l'article se pose comme objectif d'analyser la nature de la relation qui unit un élément iconique à un élément linguistique constituant l'essentiel du message transmis par les mèmes Internet. En deuxième lieu, il faut s'interroger sur la nature de la diffusion de mèmes Internet humoristiques dans le cadre de l'analyse traductologique.

\section{MÈMES DANS LA CYBERCULTURE - RIRE COMMUNAUTAIRE}

La spécificité de la cyberculture repose sur la technologie, bien sûr, mais aussi sur la création des nouvelles formes de communication s'appuyant, dans leur majeure partie, sur une image au sens large du terme, sur un élément iconique statique ou dynamique, riche ou simplifié, rendant l'interaction en ligne possible, efficace et amusante. Les formes de communication non verbale au sein de l'Internet, ont contrarié les théories créées au début de l'étude de ces phénomènes numériques qui prônent l'idée que la technologie limite l'interaction et que le manque d'un face à face ne peut pas être compensé. Le contact par Internet était considéré comme insuffisant, n'étant pas capable de se transformer en relation vraie et émotionnelle (Kozinets, 2012). La communication d'Internet, composée de codes visuels, auditifs et verbaux, se trouve quelque part entre l'oralité et l'écriture et entre le réel et le virtuel. Elle se réalise dans l'interaction qui, bien qu'elle manque de contacts directs entre les internautes, se caractérise par les aspects des échanges oraux. L'une des caractéristiques de la communication orale est son caractère coordonnant et agrégatif qui se manifeste par le fait que les interlocuteurs construisent leurs énoncés en ajoutant successivement des éléments (ŻydekBednarczuk, 2004). La communication Internet est également marquée par des émotions, donc son caractère est emphatique et participatif, plutôt qu'objectif et distancié (Ong, 2002). Cette interaction s'inscrit dans le type de communication cognitivo-affective qui se construit un nouveau modèle de signes verbo-visuels (Wagener, 2020).

La création d'Internet et de ses différentes applications est l'environnement idéal pour la propagation rapide et à grande échelle des mèmes Internet. En outre, la flexibilité du réseau, son ubiquité et son accès facile permettent aux internautes de transfor- 
mer des mèmes et d'en créer des nouveaux très facilement. Les utilisateurs vont alors partager des textes déjà existants dans un monde pré-Internet, mais aussi et surtout un nouveau genre d'expression spécifique au Web, celui des mèmes Internet. Ces derniers se décrivent comme étant une idée propagée en masse sur le web et détenant une touche humoristique. Prenant naissance sous plusieurs formes, soit par image, hyperlien, vidéo, ou encore par un personnage apparaissant à de nombreuses reprises, ceux-ci sont partagés par le biais des réseaux sociaux ou par des sites web spécifiques diffusant du contenu viral. Une définition du mème Internet a été proposée, entre autres, en 2009 par Patrick Davison dans The Language of Internet Memes : « [...] is a piece of culture, typically a joke, which gains influence through online transmission » (Davison, 2012, p. 122). L'acception plus générale de cette notion décrit le mème comme une idée, un comportement ou un style qui se transmet d'une personne à une autre à l'intérieur d'une même culture. Les mèmes sont décrits comme des unités de transmission culturelle qui se propagent par imitation. Plus particulièrement, ce terme est employé pour décrire une assimilation et une propagation rapides des unités culturelles sous forme d'images, de textes et de vidéos (Shifman, 2013). Le point de départ pour la création du mème est constitué par un élément visuel, une image complétée par un texte étant sa composante verbale. Ce qui définit le fonctionnement de cette forme de communication, c'est le fait que le mème joint des variantes soit par le contenu, soit par la forme ou encore par le point de vue de son créateur.

Les deux caractéristiques propres au mème sont la récurrence et l'aspect communautaire. L'objectif de la publication d'un mème sur Internet est de diffuser une blague verbo-visuelle jusqu'à ce que l'effet comique disparaisse du fait de la répétition accrue de cette dernière, ce qui réfère au phénomène de la récurrence. L'aspect communautaire, quant à lui, est déterminé par la rapidité avec laquelle le mème se propage atteignant ainsi le plus grand nombre d'internautes. Les mèmes Internet sont considérés soit comme une forme de culture en elle-même, soit comme étant la culture de référence pour la communauté de l'Internet, puisque leur force réside dans la création de nouvelles références. Ce phénomène viral s'est transformé petit à petit en un nouveau mode de langage utilisé couramment par les internautes dans le but de communiquer des contenus humoristiques.

\section{CRÉATION DES MÈMES HUMORISTIQUES - ENTRE IMAGE ET TEXTE}

Les mèmes sont devenus une forme prototypique de la communication humoristique sur Internet. La communauté d'internautes a créé sa propre façon de transmettre des contenus humoristiques qui profitent des possibilités de cette cybercommunauté. Malgré toute sa richesse formelle et sa diversité fonctionnelle, l'humour se cristallise le plus souvent, dans la communication réelle, sous une forme privilégiée, celle 
d'une histoire drôle dans une communauté réelle ou celle d'un mème dans l'espace virtuel. Pour les mèmes ce lieu de diffusion est défini comme un cyberespace, des communautés concrètes, liées à certains forums et certains sites internet. Le cyberespace (donc l'espace numérique, virtuel) est traité comme l'espace physique : les internautes cherchent un «lieu » concret, un forum, un blog et ils y échangent des mèmes. L'espace numérique, contrairement à l'espace physique, traditionnel, n'a ni limites ni frontières, ses début et fin sont fluctuants, il est privé de concrétisation de lieu, ses couches interfèrent et glissent. Ses utilisateurs le reconstituent continuellement (Szpunar, 2008). C'est une communauté difficile à définir, qui embrasse tous les internautes connaissant les mêmes codes, les mêmes images et citations, qui partagent leurs idées dans plusieurs sites Internet. C'est une communauté internationale, composée principalement de membres de classes sociales très variées. Il faut souligner que les limites de la cybercommunauté sont aussi fluentes que les limites du cyberespace. Les mèmes fonctionnent selon leur capacité à mobiliser des références partageables et aisément reproductibles, tout en réussissant à faire cristalliser des sensations ou des pensées dans une unité texto-iconique qui représente un nouveau type de langage, avec ses propres signes (comprenons par-là ses propres signifiés et ses propres signifiants). Le mème constitue, donc, un signe, une unité de base de la communication Internet ayant à la fois les fonctions affective et cognitive. Il est directement lié à la créativité des internautes et aux phénomènes sociaux qui englobent l'actualité et la culture pop en les croisant avec une distance humoristique ou critique. Comme signes de la communication virtuelle, les mèmes Internet constituent, selon Wagener (2020) les vecteurs du processus de sémiose numérique en créant des « nœuds de connexion » grâce auxquels les interactants forment et interprètent le sens.

Nous pourrions retrouver une certaine analogie au niveau de la création d'un effet humoristique, entre le mème et l'histoire drôle. Selon la théorie de l'humour d'Attardo, l'histoire drôle représente une structure privilégiée d'humour verbal. Raconter une histoire drôle, ou diffuser un mème Internet, le cas échéant, d'après Attardo (1994, p. 320), est une situation prototypique de l'humour verbal ou verbo-visuel : « Joke telling is the simplest and most common humorous interaction. It is the prototypical situation of linguistic humour ». Les mèmes Internet qui jouent dans le monde virtuel le même rôle que les histoires drôles dans la communication réelle, utilisent tous les éléments considérés par telle ou telle communauté comme producteurs du comique, en ayant le potentiel de provoquer le rire. Dans la communication Internet, le discours humoristique existe parallèlement à d'autres types de discours, représentant la face du divertissement, du jeu de créativité, de la gratuité à côté du sérieux de la communication standard.

C'est un discours qui s'organise autour des structures linguistiques propres au comique, comportant des allusions explicites ou implicites. La source du comique dans les mèmes réside dans la disproportion entre valeurs, structures, conventions, formulations et la réalité de référence. Le principe constitutif de l'humour (celui de disproportion ou d'anomalie selon la théorie du comique d'Émelina) constitue la raison 
d'être de ce type de discours humoristique et représente également un élément essentiel dont le rôle est d'attirer l'attention et l'intérêt du récepteur en exigeant de sa part une interprétation selon le code culturel, social et l'expérience personnelle de celui-ci. Parmi les traits sémantiques attribués habituellement aux mèmes, il faut énumérer la dissociation, le caractère fictif et hyperbolique du récit et l'attente (créée explicitement ou implicitement auprès du public) comme une condition sémantique de réception.

En parlant du sémantisme des mèmes, il faut souligner que la disproportion ou la dissociation entre la réalité, représentée par la composante iconique du mème, et la manière de la présenter par celui-ci peut s'opérer à plusieurs niveaux. La communication virtuelle a élaboré sa forme humoristique homologue, celle du mème Internet qui en reprenant la structure narrative de l'histoire drôle, en remplace une partie par une image. Le mème Internet constitue donc une forme iconique-verbale dans laquelle l'image joue un rôle primordial par rapport à l'élément verbal. Le mème est créé par le processus d'interprétation au deuxième degré c'est-à-dire intentionnelle et indirecte, qui a pour but de créer un effet humoristique. L'internaute, créateur des mèmes qui circulent dans le cyberespace, utilise un élément iconique qui représente un potentiel humoristique, dans le sens de la théorie d'Attardo, donc qui se laisse emprunter dans le mécanisme humoristique par l'ajout d'un élément verbal qui assume un rôle de disjoncteur humoristique ${ }^{1}$. Ainsi, le mème se crée-t-il sous la forme d'un amalgame conceptuel d'idées transmises par deux types de codes : visuel et verbal. La notion d'amalgame, en tant que principe ontogénétique et processus linguistique, parfois défini en termes cognitifs, est perçue comme un phénomène d'hybridation formelle et sémantico-cognitive de l'interprétation d'énoncés. En revanche, l'amalgame est reconnecté à l'humour et à l'analogie. Il est envisagé comme un processus cognitif, sans prise en compte des affects, et opérant au-delà du domaine des formes linguistiques (Fortis, Col, 2018). Il convient de mentionner la notion de frame blend qui définit l'humour comme le fruit d'une rencontre entre univers ou situations. Cette perspective remonte en fin de compte à l'analogie d'Aristote : un frame blend est une espèce d'analogie entre situations, mais dans l'humour cette analogie est incomplète. Dans l'histoire qu'on lit ci-après, c'est précisément le caractère incomplet de l'analogie qui suscite l'amusement. L'interprétation, ou traduction au sens d'explication, s'avère métonymique : de toute la richesse de possibilités interprétatives, le créateur de ce mème utilise le concept de tristesse suggéré par la photo pour y joindre un commentaire qui renvoie à un problème banal et marqué culturellement dans la société polonaise. Le caractère incomplet de l'analogie se manifeste entre deux schémas logiques de cause à effet encodés dans deux ordres : l'un visuel et l'autre verbal. À l'image, il lui manque une cause qui reste présupposée et nous fait penser à un problème très grave. Pourtant, elle s'avère banale dans le texte ajouté qui déclenche un effet humoristique.

${ }^{1}$ Dans la théorie de script d'Attardo, le disjoncteur humoristique est défini comme un élément sémantique ou référentiel qui actualise le potentiel comique introduit par le connecteur dans un récit humoristique. 


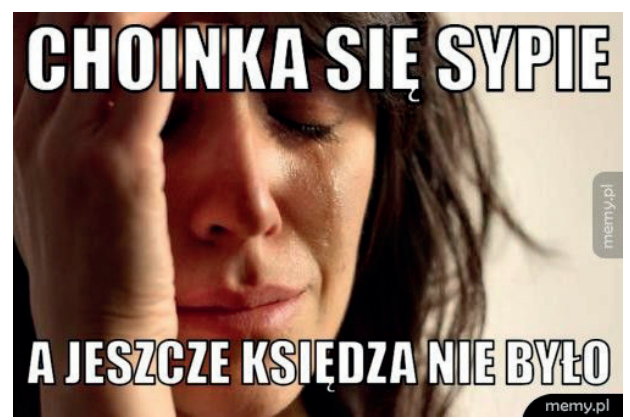

Fig. 1. Jak żyć ? (Comment vivre ?)

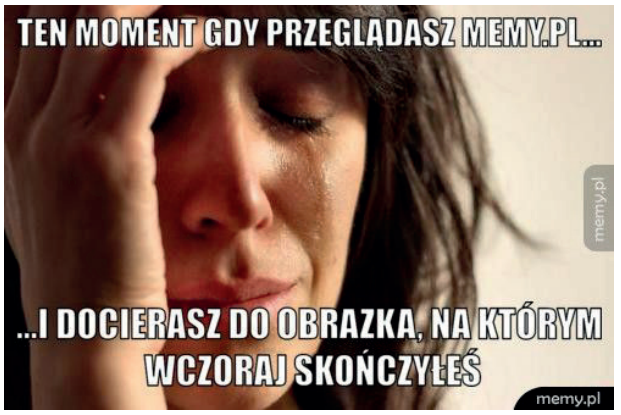

Fig. 2. Ten moment gdy... (Le moment où...)

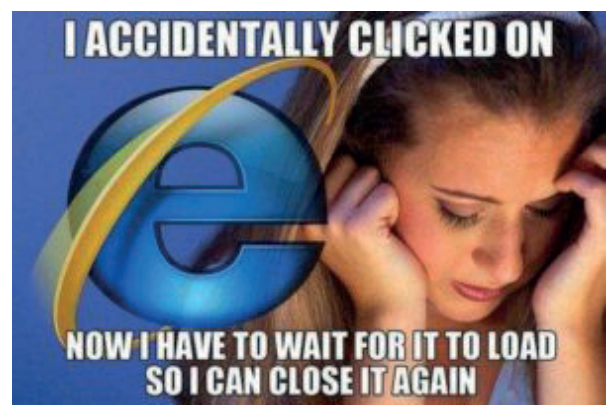

Fig. 3

Nous pourrions facilement nous imaginer la même image dans la même série de mèmes (le type : Comment vivre ? Le moment où...) mais avec un texte-réponse différent et formulé dans une autre langue, diffusé par un internaute traducteur-créateur qui participe activement dans la communication sur Internet tout en étant à la fois récepteur et producteur de messages humoristique. La diffusion de différentes versions conceptuelles ou linguistiques (au niveau d'un composant linguistique) du mème peut-elle être traitée comme une forme de traduction?

When c'est la fin du mois

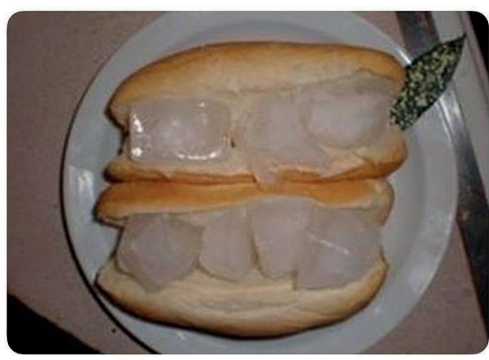

Fig. 4 
Le mème représente une unité sémantico-formelle indissociable qui est de nature hétérogène car il unit deux types de codes qui forment un signe iconico-textuel fonctionnant dans la communication virtuelle comme un nouveau langage dont les signes sont de forme mixte. Ce signe se comporte comme les signes de la même nature, par exemple, les signes linguistiques qui sont échangeables dans les calambours. Le mème ci-dessous illustre ce type de jeux de mots, le cas échéant, un jeu de mot et d'image. L'élément verbal 'Barack' se visualise dans le portrait qui, par sa stylistique, se verbalise dans 'Baroque' en créant un effet humoristique.

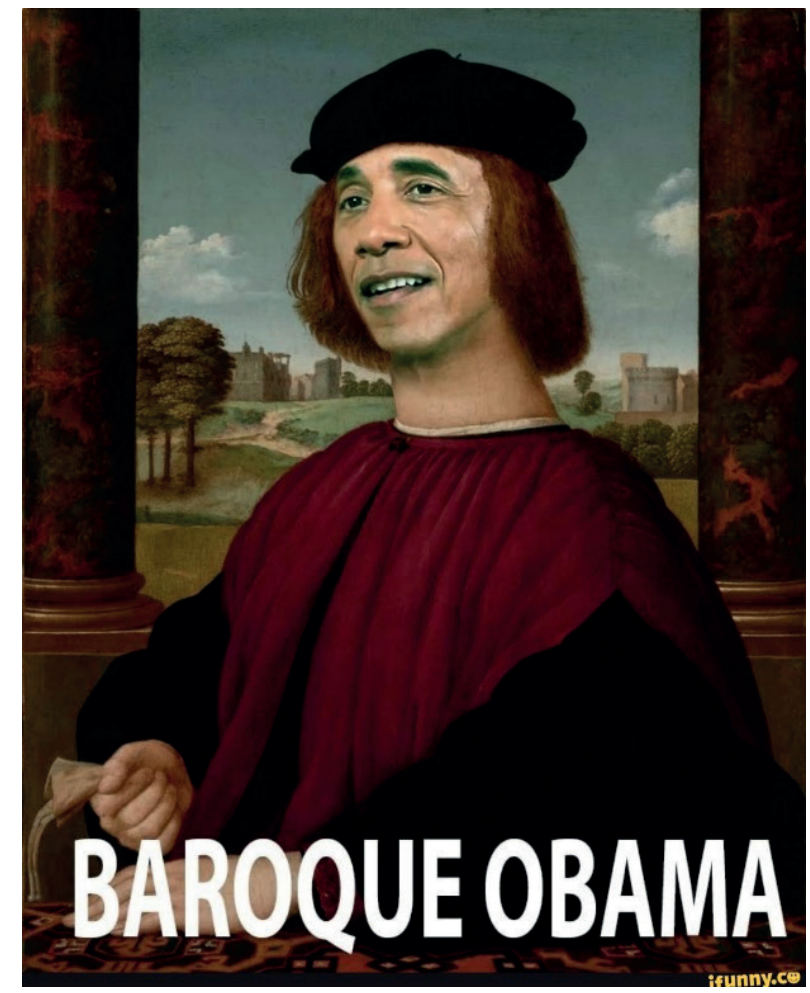

Fig. 5

Les fonctions identitaires de 1'humour ont fait 1'objet d'analyses chez Gasquet-Cyrus (2002). Ce dernier s'appuie dans son approche sur le concept du rire stéréotypé de Victoroff (1953) qui repose sur les représentations collectives et les normes en circulation dans une société, une culture ou une communauté. L'exemple suivant (Fig. 6) illustre un type d'humour situationnel (nommé par Victoroff le rire spontané) dans lequel le texte constitue un commentaire de la situation présentée par le mème mais renvoyant au-delà du monde virtuel, c'est-à-dire à l'actualité politique. 

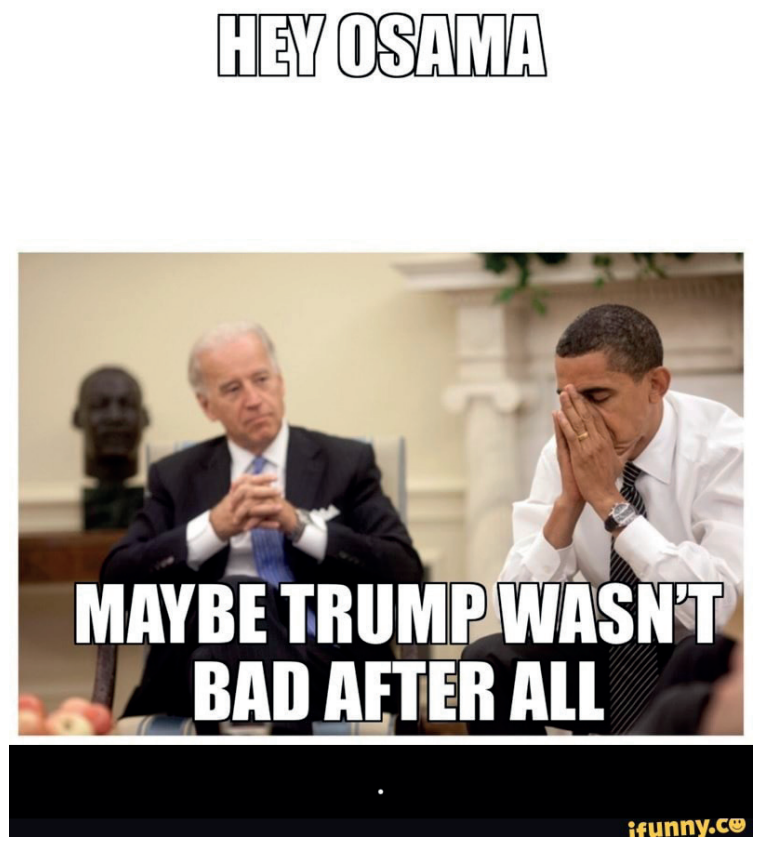

Fig. 6

Au niveau des représentations collectives, l'humour semble fonctionner comme le langage. Dans les interactions verbales, chaque communauté possède ses moyens verbaux, para-verbaux ou non-verbaux pour marquer le changement de registre, qui préparent les participants de l'interaction, au niveau cognitif et affectif, à la réception d'un récit humoristique. Ces éléments préparatifs rendent l'échange humoristique possible et efficace. Sans avoir créé chez le récepteur l'attente de la réception d'une forme ludique, l'effet humoristique de celle-ci ne se concrétise pas.

Le rôle de ce type d'énoncés, qui apparaissent dans une conversation, consiste à restructurer les fonctions des participants de l'échange. Ils créent de la place dans l'échange que l'on est en train de réaliser, pour y insérer un autre type de discours. En plus, les participants, qui jusque-là assumaient, à parts égales, la responsabilité cognitive et affective de la réussite de l'échange, deviennent respectivement narrateur/créateur et récepteur, en prenant leurs distances par rapport à ce discours qui va leur être présenté. Le jeu commence, on en connait la règle : celle d'en saisir et d'en apprécier le comique. Dans le discours humoristique de la communauté d'internautes, le mème, par sa fonction et sa forme, oriente l'interprétation du message véhiculé. Ainsi, le mème devient une forme prototypique de la communication humoristique sur Internet.

Ce type de marquage humoristique apparaît dans la réflexion théorique de Gumperz (1982) qui parle de contextualization cues (appelés aussi marques de contextuali- 
sation, déclencheurs humoristiques, ang. ambiguators, katalizatory dwuznaczności²). Ces éléments discursifs qui renvoient au savoir contextuel des interlocuteurs permettent de situer le message d'une manière adéquate parmi d'autres énoncés et d'en interpréter l'intention. L'importance de ces catalyseurs humoristiques pour l'interprétation de l'intention de l'émetteur du message est soulignée aussi dans les travaux de Nash (1985), de Mulkay (1988) et de Norrick (1993). La structure linguistique des manifestations humoristiques, par exemple des histoires drôles ou des mèmes Internet, renferme déjà une méta-information sur leur caractère humoristique (Attardo, Chabanne, 1992).

Les conventions pragmatiques ne concernent que la forme elle-même des mèmes humoristiques, la cyberculture a créé les propres conventions de leur création et de leur réception. L'attente réceptive créée chez le récepteur s'inscrit dans la notion d'esthétique de la réception et implique l'existence chez le récepteur de connaissances préexistant à un échange concret sur les structures, le fonctionnement et les contenus du discours humoristique sur Internet. Les marqueurs de changement de niveau qui annoncent une blague signalent le passage du parler référentiel, direct, au parler fictionnel/fictif, indirect.

Au niveau social, l'une des fonctions essentielles de l'humour est d'ordre identitaire. Le sentiment d'appartenance à une communauté est toujours très fort et il se manifeste aussi dans le langage, dans les formes d'interactions iconico-verbales réalisées par le groupe et par le type de discours élaboré par un groupe donné. La notion de discours comique/humoristique s'inscrit aussi dans le domaine consacré à l'étude de communautés discursives qui élaborent, en fonction de leurs conditions socioculturelles, des genres textuels utilisés dans la communication.

\section{TRANSFERT DE MÈMES DANS LE MILIEU MULTILINGUE DE LA CYBERCULTURE}

Le fonctionnement des mèmes Internet suscite aussi une question d'ordre traductologique portant sur la nature de la propagation de cette forme humoristique dans le cyberespace. Toutes les tentatives de définition du type de transfert de mèmes qui s'opèrent dans la communication humoristique sur Internet doivent obligatoirement prendre en considération les paramètres suivants :

- les caractéristiques formelles et conceptuelles du texte de départ (la notion de texte prise au sens large du terme : celui du texte de culture). Le mème

${ }^{2}$ Lew (2000, pp. 127-135) parle de trois fonctions principales de katalizatory dwuznaczności dans l'interprétation des manifestations de l'humour verbal : la première fonction consiste à limiter la probabilité de l'interprétation non marquée humoristiquement, la deuxième a pour but de renforcer l'interprétation marquée et la dernière fonction s'apparente au rôle des éléments introducteurs qui préparent l'appareil interprétatif des interlocuteurs à la perception et à la compréhension d'un autre type de discours. 
Internet constitue donc un message iconico-verbal à caractère humoristique qui symbolise des concepts, des unités de cultures. Vu les caractéristiques du message de départ, le transfert de mèmes se situe du côté de la traduction intersémiotique (Tomaszkiewicz, 2010) ;

- le milieu de fonctionnement qui détermine la manière dont se propage un texte, incluant les paramètres techniques tels que : le support technologique (les multimédia, Internet), la communication dans le monde virtuel, nous renvoie, plutôt à la traduction multimédias ;

- les caractéristiques linguistiques et culturelles de la communauté au sein de laquelle se réalise le transfert. Celle-ci fonctionne dans la cyberculture en se créant un nouveau langage en commun composé d'éléments linguistiques appartenant à des langues différentes.

Le mème Internet représente, sans aucun doute, une forme de macro-signe hétérogène au niveau de codes en unissant des éléments visuels (plus ou moins détaillés : à partir des dessins assez schématiques jusqu' aux photos bien artistiques) à des signes linguistiques. Cet amalgame multisémiotique enferme un contenu conceptuel basé sur un fond culturel propre aux internautes qui sont à la fois créateurs et récepteurs de ce discours. Le fonctionnement des mèmes puise dans le fond commun de la cyberculture qui s'enrichit grâce au partage des cultures individuelles des internautes qui également désignent les règles discursives de création, réception et transmission de mèmes. Ceux-ci tout en restant au sein de la cyberculture commune font partie de la communication multilingue étant donné que les internautes représentent des communautés linguistiques très diverses. La participation à la culture, soit passive comme récepteur de celle-ci, soit active comme créateur, relève de la compétence culturelle acquise.

Si l'on définit le mème comme une unité de culture fonctionnant dans le discours humoristique sur Internet, il faut souligner que les formes de transmission sont gérées par deux mécanismes principaux : ceux d'imitation et de modification, ceux de création et de réinterprétation. Ce dernier processus aboutit à la création de plusieurs variantes d'un mème donné unies soit par un concept commun, soit par le même élément visuel repris avec un autre commentaire ou encore par le même mécanisme générateur de l'humour qui unit le verbal à l'image. Ce lien, dans les mèmes humoristiques, s'appuie sur une relation d'interprétation (Tomaszkiewicz, 2010, p. 39) à des fins humoristiques, donc, nécessairement contradictoire à l'intérieur du même macro-signe.

Ce qui est à souligner étant donné le cadre traductologique de cette étude, c'est l'une des caractéristiques de la culture décrite par Samovar et Porter (1997) celle du caractère transmissible de la culture donc du comique. Il en résulte que l'effet humoristique, pour qu'il soit atteint, exige une expérience commune, un vécu social et culturel partagé. Autrement dit, celui qui perçoit un message humoristique (locuteur, auditeur ou lecteur) doit avoir à peu près le même système de représentations mentales et d'évaluations de l'univers référentiel. 
La notion d'humour est fortement liée à celle de langue-culture présente dans les études ethnolinguistiques surtout par son ancrage dans la réalité culturelle et sociale des usagers de la langue. Cet ancrage est visible à plusieurs niveaux :

- au niveau verbo-visuel, sous forme de connotations sémantiques encodées dans le lexique et le visuel ;

- au niveau discursif, par une organisation spécifique du discours humoristique, par des éléments situationnels de production et de perception de celui-ci ;

- au niveau interactionnel, dans les principes qui sous-tendent le fonctionnement des conversations des différents types d'échanges communicatifs, entre autres ceux où apparaît un élément comique.

Le terme de langue-culture, le cas échéant langue-cyberculture, recouvre entre autres la notion de représentations partagées qui renvoient à des mobilisations, généralement implicites, en discours, d'un sens plus ou moins commun, représentations intra- et intercommunautaires à teneur plus ou moins clairement normative et présentant un degré de figement plus ou moins important, qui se manifestent dans des évaluations, des catégorisations, des allusions traditionnelles à la mémoire, dans sa majeure partie iconique, de la cyberculture. Le mème unit deux types de signes dont le degré d'iconicité est différent : l'un, en étant iconique, renvoie directement à une représentation mentale d'un référent réel facilement accessible, l'autre symbolique, un signe linguistique, dont la signification n'est pas immédiatement saisissable.

\section{CONCLUSION}

Les images sont omniprésentes dans notre société. Et le rapport entre image et texte n'est pas anodin. Est-il besoin de dire que la traduction d'un texte associé à des images ne peut faire abstraction de ces dernières ? Cet article sur l'interaction image-texte-traduction/ diffusion montre que leurs rôles respectifs dans la communication sur Internet ne sont pas les mêmes que dans la communication traditionnelle dans le monde réel. Dans l'espace virtuel, la communication dans sa majeure partie est ancrée dans le visuel qui constitue sa raison d'être. Cette interaction particulièrement étroite entre texte et image pose souvent des problèmes quand il s'agit de la définition du processus de diffusion de mèmes dans un milieu multilingue mais, à la fois, disposant de mêmes références dans la cyberculture.

Du côté des internautes, les mèmes humoristiques contribuent à l'expression de soi et à la création de communautés. Ces deux phénomènes faisant partie de la dilatation sémiotique d'un univers virtuel. La diffusion de mèmes se réalise par un acte de réinterprétation créative intralinguale de ces macro-signes qui constituent le système langagier de la communication dans le cyberespace. La facette visuelle du mème, inséparable de sa composante linguistique, garantit l'instantanéité de sa perception au niveau formel et conceptuel par les internautes. La saisie du message est immédiate, 
grâce à sa composante visuelle, mais elle n'épuise pas la totalité de ses possibilités interprétatives au sein de ce discours humoristique dans le monde virtuel, d'où le déclenchement de l'étape suivante, celle de la création de nouveaux mèmes. Ceci se fait par la modification, la reconceptualisation d'une des composantes de ce signe iconico-verbal : visuelle, linguistique ou conceptuelle. Le processus de sémiose dans ce langage virtuel se manifeste par une recontextualisation et une reformulation de mèmes qui contribuent à leur diffusion par le biais du processus de retraduction interprétative dans la communication virtuelle. 


\section{BIBLIOGRAPHIE}

Attardo, S. (1994). Linguistic Theories of Humor. In V. Raskin, M. Apte (eds.), Humor Research 1. Berlin/ New York : Mouton de Gruyter.

Attardo, S. (2001). Humorous Texts. A Semantic and Pragmatic Analysis. In V. Raskin, W. Ruch (eds.), Humor Research, 6. Berlin/New York : Mouton De Gruyter.

Attardo, S. (2002). Translation and Humour : An Approach Based on the General Theory of Verbal Humour (GTVH). Manchester : St. Jerome Publishing.

Attardo, S., Chabanne, J. (1992). Jokes as a text type. Humor: International Journal of Humor Research, $5(1-2), 165-176$.

Baran, A. (2012). Visual humour on the Internet. In Estonia and Poland: Creativity change in cultural communication. Vol. 1: Jokes and humour (pp. 144-158). DOI: doi.org/10.7592/EP.1.baran.

Blackmore, S. (1999). The Meme Machine, Oxford University Press, trad. française (2006) : La théorie des mèmes. Pourquoi nous nous imitons les uns les autres, trad. B. Thomass, Paris : Max Milo Éditions.

Davison, P. (2012). The Language of Internet Memes. In M. Mandiberg (ed.), The Social Media Reader. URL : https// veryinteractive.net/library/the-language-of-internet-memes.

Dawkin, R. (1976). The Selfish Gene. Oxford : Oxford University Press. DOI : 10.4324/9781912281251.

Émelina, J. (1996). Le comique. Essai d'interprétation générale. Paris : Éd. SEDES.

Fortis, J.-M., Col, G. (2018). Espaces Mentaux et Intégration Conceptuelle : Retour sur la Constitution de Théories Sœurs. Revue de l'Association française de linguistique cognitive. CogniTextes (en ligne), 18. DOI : https ://doi.org/10.4000/cognitextes.1111.

Gasquet-Cyrus, M. (2002). Pour une étude sociolinguistique de l'humour : l'humour marseillais. In M. Madini (ed.), 2000 ans de Rire. Permanence et Modernité, Coll. «Linguistique et sémiotique » 42 (pp. 251-259). Besançon : PU Franc-Comtoises.

Gumperz, J. (1982). Discourse Strategies. Cambridge : Cambridge University Press.

Kozinets, R.V. (2012). Netnografia. Badania etnograficzne online (trad. de M. BrzozowskaBrywczyńska). Warszawa : PWN.

Lew, R. (2000). Dowcip językowy w świetle najnowszych językoznawczych teorii humoru. In S. Gajda, D. Brzozowska (eds.), Świat humoru (pp. 127-135). Opole: Wydawnictwo Uniwersytetu Opolskiego.

Mulkay, M. (1988). On Humor. Its Nature and its Place in Modern Society. Cambridge : Blackwell.

Nash, W. (1985). The Language of Humour: Style and Technique in Comic Discourse. London/New York : Longman.

Norrick, N.R. (1993). Conversational Joking. Humor in everyday talk. Bloomington, Indianapolis : Indiana University Press.

Ong, W.J. (2002). Orality and Literacy: The Technologizing of the Word ( $2^{\text {nd }}$ ed.). New York : Routledge.

Samovar, L.A., Porter, R.E. (eds.) (1997). Intercultural Communication. New York/Wadsworth : Peter Lang.

Shifman, L. (2013). Memes in a digital world: Reconciling with a conceptual troublemaker. Journal of Computer-mediated Communication, 18, 362-377. DOI : 10.1111/jcc4.12013.

Szpunar, M. (2008). Redefinicja pojęcia czasu i przestrzeni w dobie Internetu. Kulturowe kody mediów. Stan obecny i perspektywy rozwoju, 59-73.

Tomaszkiewicz, T. (2010). Przekład audiowizualny, werbo-wizualny czy intersemiotyczny: różne wymiary tej samej rzeczywistości? Lingwistyka Stosowana, 3, 33-44.

Victoroff, D. (1953). Le rire et le risible. Introduction à la psycho-sociologie du rire. Paris : PUF.

Wagener, A. (2020). Mèmes, gifs et communication cognitivo-affective sur Internet. Communication (en ligne), 37/1. URL : http:// journals.openedition.org/communication/11061. DOI : http://doi. org/10.4000/communication.11061. 
Yus, F. (2018). Identity-Related Issues in Meme Communication. Internet Pragmatics, 1(1), 113-133. DOI : 10.1075/ip.00006.yus.

Zaremba, M. (2012). Memy internetowe (20102011). Media i Społeczeństwo, 2.

Ziv, A. (1987). Le sens de l'humour. Paris : Bordas.

Żydek-Bednarczuk, U. (2004). Tekst w Internecie i jego wyznaczniki. In M. Kita (ed.), Dialog a nowe media (pp. 11-21). Katowice : Wydawnictwo Uniwersytetu Śląskiego.

Sources des mèmes

http://ifunny.co

http://memy.pl 